\title{
Role played by edge-defects in the optical properties of armchair graphene nanoribbons
}

Thi-Nga Do ${ }^{1}$, Godfrey Gumbs ${ }^{2}$, Danhong Huang ${ }^{3}$, Bui D. Hoi ${ }^{4}$, Po-Hsin Shih ${ }^{1 a}$

${ }^{1}$ Department of Physics, National Cheng Kung University, Tainan 701, Taiwan

2 Department of Physics and Astronomy,

Hunter College of the City University of New York,

695 Park Avenue, New York, New York 10065, USA

${ }^{3}$ US Air Force Research Laboratory,

Space Vehicles Directorate (AFRL/RVSU),

Kirtland Air Force Base, New Mexico 87117, USA

${ }^{4}$ Department of Physics, University of Education,

Hue University, Hue 530000, Viet Nam

(Dated: October 24, 2021)

\footnotetext{
${ }^{a}$ Corresponding author: E-mail: phshih@phys.ncku.edu.tw
} 


\begin{abstract}
We explore the implementation of specific optical properties of armchair graphene nanoribbons (AGNRs) through edge-defect manipulation. This technique employs the tight-binding model in conjunction with the calculated absorption spectral function. Modification of the edge states gives rise to the diverse electronic structures with striking changes in the band gap and special flat bands at low energy. The optical-absorption spectra exhibit exotic excitation peaks and they strongly depend on the type and period of the edge extension. Remarkably, there exist the unusual transition channels associated with the flat bands for selected edge-modified systems. We discover the special rule governing how the edge-defect influences the electronic and optical properties in AGNRs. Our theoretical prediction demonstrates an efficient way to manipulate the optical properties of AGNRs. This might be of importance in the search for suitable materials designed to have possible technology applications in nano-optical, plasmonic and optoelectronic devices.
\end{abstract}

\title{
I. INTRODUCTION
}

Seventeen years have now passed since the discovery of graphene in 2004 [1] and this has unmistakably inspired a huge amount of research on its fundamental properties as well as those of graphene-related materials. Graphene nanoribbons (GNRs), which are narrow strips of graphene, possess quasi-one-dimensional properties. This material presents rich essential physical properties, including electronic, optical, magnetic, and transport properties. [2-7] Consequently, they acquire additional advantages over graphene sheets in the point of view of certain technological applications such as nanoelectronics, spintronics, and photodetectors. [8-10] Up to now, the bottom-up [11-15] and top-down [16-20] methods have been demonstrated to be efficient for the synthesis of GNRs. It is well known that GNRs can be classified into two categories based on their edge-state arrangement, namely, armchair GNR (AGNR) and zigzag GNR (ZGNR) [21, 22]. Both the AGNR and ZGNR can be subjected to edge defects during the fabrication processes, which has been examined to modify remarkably the essential physical properties of the materials. This work will mainly focus on the role played by the edge-extension on the electronic and optical characteristics of AGNRs.

In previous studies, it has been demonstrated that fundamental physical properties of 
GNRs can be tailored by the presence of edge defects [13, 23-25], besides edge terminations, ribbon width, and applied electrostatic fields [2-5]. The edge-defect GNRs with proper configuration have been demonstrated as being suitable for certain device applications, such as bandgap engineering for generating semiconductor heterostructure devices [12, 16], improved electrical conductivity for efficient gas sensing [13], and imbalanced sublattice for creating graphene nanomaterials with magnetically nontrivial ground states [15]. Recently, the band structures and charge-density distribution of AGNRs have been predicted theoretically as well as examined experimentally to be significantly responsive to the kinds of edge modification [23]. Various types of electronic topological phases and the influence of electronic correlations on these topological states have been reported [24, 25]. Despite recent progress in this field, the investigation of the optical properties of edge-extended GNRs is still in its infancy. Consequently, engineering the optical spectra of GNRs by introducing edge defect is worthy of a careful investigation.

In this paper, we carefully analyze the electronic and optical properties of AGNRs with various types of edge extensions. The unique absorption spectra and their relations to the special electronic structure, including the tunable band gap and flat bands, will be discussed extensively. The density of states (DOS), which is crucial for an in depth understanding of both the electron state distribution and optical excitation channels, are presented. Our numerical calculations reveal unusual optical selection rules related to the transition of the nearly-flat edge bands. We demonstrate that the frequency and amplitude of the opticalabsorption peaks can be efficiently manipulated by both the type and period of the edge extension.

The rest of this paper is organized as follows. In Sec. II, we present our theoretical method which we use for executing our numerical computations of the properties of edge-extended AGNRs. Section III contains a detailed discussion of the band structure and absorption spectra for the systems with three different kinds of edge-extension. The principal results of this work are summarized in Sec. IV.

\section{THEORETICAL METHOD}

We begin by noting that a GNR consists of carbon atoms stacked together to form a honeycomb lattice with a bond length of $b=1.42 \AA$. The tight-binding model is employed 
to investigate the electronic and optical properties of AGNR. The couplings between nearestneighbor atoms are included in our calculations. For pristine AGNR along the $\mathrm{x}$ direction, the width $(\mathrm{N})$ is estimated by the number of carbon atoms across the ribbon along the $\mathrm{y}$ axis. Here, we consider the AGNR with extended segments being added periodically on both sides along the $\mathrm{x}$ direction. Figures 1(a) through 1(c) illustrate respectively three different configurations of edge-extended AGNRs; they are 7/9-AGNR heterojunction (J$(n, m))$, staggered edge-extended AGNR $(\mathrm{S}-(n, m))$, and inline edge-extended AGNR (I$(n, m))$. In this notation, $n$ and $m$ stand for the length of the edge-extended and original segments, respectively. They are integers in the unit of $3 b$. A primitive unit cell comprises two continuous $n$ and $m$ segments. For $\mathrm{J}_{-}(n, m)$ and $\mathrm{S}-(n, m)$, the two component segments possess the widths of $N$ and $(N+2)$ while they are $N$ and $(N+4)$ for I- $(n, m)$. Furthermore, the two edges of $\mathrm{J}-(n, m)$ and $\mathrm{I}-(n, m)$ are equivalent along the backbone of the ribbon. On the other hand, the edge-extended segments are located alternately on each side of the $\mathrm{S}-(n, m)$ AGNR. The new boundary condition related to each type of edge-extension is taken into consideration by the proper modification of the Hamiltonian. The difference in configuration among these AGNRs gives rise to their distinctive electronic and optical characteristics.

The low-energy electronic properties of an edge-extended AGNR can be described in terms of the $p_{z}$-orbital tight-binding Hamiltonian, which we write as

$$
H=\sum_{\langle i, j\rangle} \gamma^{R_{i j}} C_{i}^{\dagger} C_{j}+\text { h.c.. }
$$

In this expression, $i$ and $j$ denote lattice sites, $C_{i}^{\dagger}\left(C_{j}\right)$ is a creation (annihilation) operator, $R_{i j}$ is the translation vector between two atoms, $\gamma^{R_{i j}}=2.6 \mathrm{eV}$ is the nearest-neighbor atomic interaction [26], and h.c. stands for the Hermitian conjugate of the first term. This Hamiltonian is properly constructed based on selected $i$ and $j$ of individual AGNR of different edge-extended types. Consequently, the corresponding band structure and wave functions automatically satisfy the boundary condition of the system.

An electromagnetic field carrying the electric polarization $\hat{\mathbf{E}}$ with frequency $\omega$ can lead to vertical optical transitions from occupied to unoccupied states in an AGNR. The resulting absorption function for vertical transition and $E_{F}=0$ can be shown to be given by [27] 

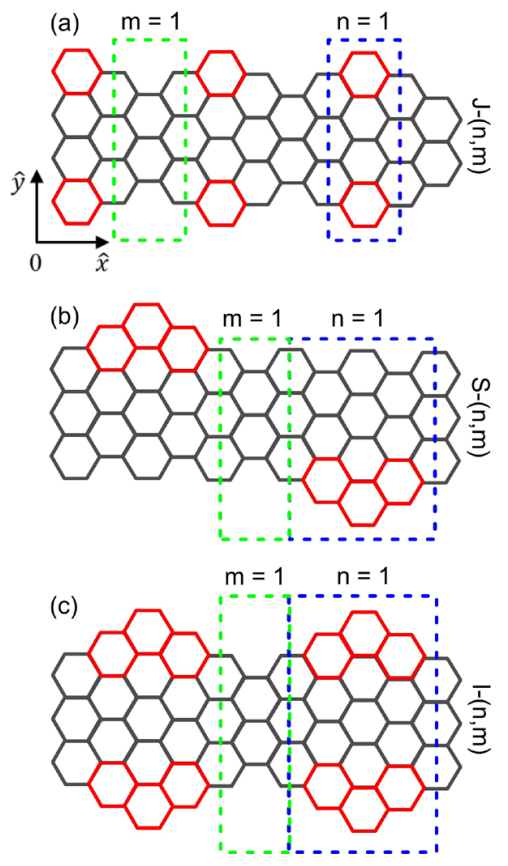

FIG. 1. (color online) Lattice structures of edge-extended AGNRs for (a) 7/9-AGNR nanoribbon, (b) staggered edge-extended AGNR, and (c) inline edge-extended AGNR. The grey hexagons denote pristine AGNRs while the red hexagons represent the edge-extended areas. The green and blue dashed rectangles indicate the units of $\mathrm{n}$ and $\mathrm{m}$ segments, respectively.

$$
\begin{gathered}
A(\omega) \propto \sum_{c, v, i, j} \int_{1 s t B Z} \frac{d \mathbf{k}}{(2 \pi)^{2}}\left|\left\langle\Psi^{c}(\mathbf{k}, j)\left|\frac{\hat{\mathbf{E}} \cdot \mathbf{P}}{m_{e}}\right| \Psi^{v}(\mathbf{k}, i)\right\rangle\right|^{2} \\
\times \operatorname{Im}\left[\frac{f\left(E^{c}(\mathbf{k}, j)\right)-f\left(E^{v}(\mathbf{k}, i)\right)}{E^{c}(\mathbf{k}, j)-E^{v}(\mathbf{k}, i)-\omega-i \Gamma}\right] .
\end{gathered}
$$

Here, $\mathbf{P}$ is the momentum operator, $m_{e}$ is the free-electron mass, $f\left(E^{c, v}(k, i)\right)$ is the FermiDirac distribution function, and $\Gamma(=1 \mathrm{meV})$ is a broadening parameter. For the absorbtion function, the available excitation channels are determined by the non-vanishing of the velocity matrix element $\left\langle\Psi^{c}\left(\mathbf{k}, m^{\prime}\right)\left|\frac{\hat{\mathbf{E}} \cdot \mathbf{P}}{m_{e}}\right| \Psi^{v}(\mathbf{k}, m)\right\rangle$. This term can be estimated by using the gradient approximation [28]. On the other hand, the absorption strength is proportional to the joint density of states $\operatorname{Im}\left[\frac{f\left(E^{c}\left(\mathbf{k}, m^{\prime}\right)\right)-f\left(E^{v}(\mathbf{k}, m)\right)}{E^{c}\left(\mathbf{k}, m^{\prime}\right)-E^{v}(\mathbf{k}, m)-\omega-i \Gamma}\right]$. 


\section{RESULTS AND DISCUSSION}

For a pristine AGNR, the band structure exhibits a band gap which is inversely proportional to the ribbon width [29]. In order to highlight the influence of the edge extension on the electronic properties of AGNRs, we introduce defects into a narrow ribbon $(\mathrm{N}=7)$. It is worth mentioning that the current experiments for the edge-defect GNRs mainly focus on the narrow GNR [14, 23]. Our numerical calculations for three different types of edge extension show that the edge bands occur in the vicinity of the Fermi level $E_{F}=0$. The valence and conduction edge bands retain the mirror symmetry. The main features of energy dispersion are sensitive to both $n$ and $m$. These characteristics are in a good agreement with the experimental measurements and previous theoretical predictions. The unique band structures are associated with the corresponding absorption spectra which we will discuss thoroughly next.

In order to investigate the roles played by $n$ and $m$ on the energy dispersion of the system, we first keep $n$ fixed but change $m$, and then keep $m$ fixed while altering the value of $n$. We find that the energy dispersion greatly depends on $n$ whereas the band gap is efficiently tuned by a factor $m$. For the heterojunction $\mathrm{J}-(n, m)$, the band structures obtained by varying $n$ and $m$ are displayed in Figs. 2(a) and 2(d). Particularly, for $n=$ 1 , the increase of $m$ gradually reshapes the $\left(v_{1}, c_{1}\right)$ pair of the bands from parabolic to nearly-flat formations, as shown in Fig. 2(a). On the other hand, the size of the band gap possesses a proportional relationship with $n$ for chosen $m$, referring to Fig. 2 (d) for $m=10$. The substantial band features and their dependence on the lattice configuration are clearly reflected in the density of states (DOS). Figures 2(b) and 2(e) illustrate the DOS where the individual curves correspond to the energy bands with the same color. There exist the dominant peaks at the energies where the extrema of the valence and conduction bands or the nearly-flat bands are located. Consequently, these peaks are symmetric with respect to the Fermi level. Their amplitudes imitate the energy dispersion, i.e., the flat bands present predominant DOS features. Furthermore, the zero DOS in the vicinity of $E_{F}=0$ indicates the absence of electronic states within the band gap. The DOS spectrum is crucial for an extensive understanding of the optical excitations via the absorption intensity, according to the absorption function in Eq. (2).

The $\omega$-dependent absorption spectra exhibit unique peaks, as demonstrated in Figs. 2(c) 
and 2(f) for various sets of $(n, m)$. Each peak corresponds to a specific transition between the extreme values of the valence and conduction bands or the nearly-flat bands. The frequency of the optical threshold $\left(\omega_{1}\right)$ reflects on the band gap. Meanwhile, its amplitude contains information regarding the state distribution and Hamiltonian matrix based on the velocity matrix elements in Eq. (2). For example, the threshold frequency of J-(1,5) (red line) is higher than that of J-(1,1) (blue line) but lower than that of J-(1,10) (green line) (see Fig. $2(\mathrm{c}))$. This is consistent with the band gap size relation for these three systems. This can also explain the difference in frequency of $\omega_{1}$ for $\mathrm{J}-(1,10), \mathrm{J}-(5,10)$, and $\mathrm{J}(10,10)$ shown in Fig. 2 (f). Moreover, the absorption peaks in the higher frequency range labeled as $\omega_{2}$, $\omega_{3}$, and $\omega_{4}$ for $\mathrm{J}-(5,10)$ and $\mathrm{J}(10,10)$ come from the transitions associated with the states at higher and deeper energies, such as $v_{2}$ and $c_{2}$ (not shown). These energy bands move towards the Fermi level for increasing $n$. Hence for AGNR, the absorption spectrum in the low-frequency region can be significantly enriched by the proper modification of the edge configuration.

The spectral intensity can be understood via the DOS. The transition between the occupied and unoccupied bands with higher-DOS is expected to yield stronger absorption intensity. This is generally true for most $\mathrm{J}-(n, m)$ systems, as illustrated in Fig. 2 (c). Interestingly, this explanation fails for specific configurations of edge-extended AGNRs, such as the J-(5,10) and J-(10,10) (see the orange and purple lines in Fig. 2 (e)). These systems present relatively low threshold optical peaks though the corresponding DOS shown in Fig. 2 (e) is predominant. We observe that the optical transition is enhanced if the initial and final states belong to the parabolic bands with opposite slopes. However, it is suppressed for the nearly-flat bands. Our numerical calculations show that, for the J-( $n, m)$ type, the Hamiltonian matrices of the systems possessing the nearly-flat edge bands contain special elements which lead to the inconsequential derivation of $\mathrm{H}$. As a result, the associated velocity matrix elements in Eq. (2) are infinitesimal. This can account for the extremely low threshold peaks of the systems with nearly-flat $v_{1}$ and $c_{1}$ edge bands. This unusual optical selection rule is attributed to the influence of edge-modification on the state distribution of the system.

For the S- $(n, m)$ AGNR, the change in energy dispersion with varying $(n, m)$ is more clear compared with that of the $\mathrm{J}-(n, m)$. For a fixed $n$, the increase of $m$ notably reduces 

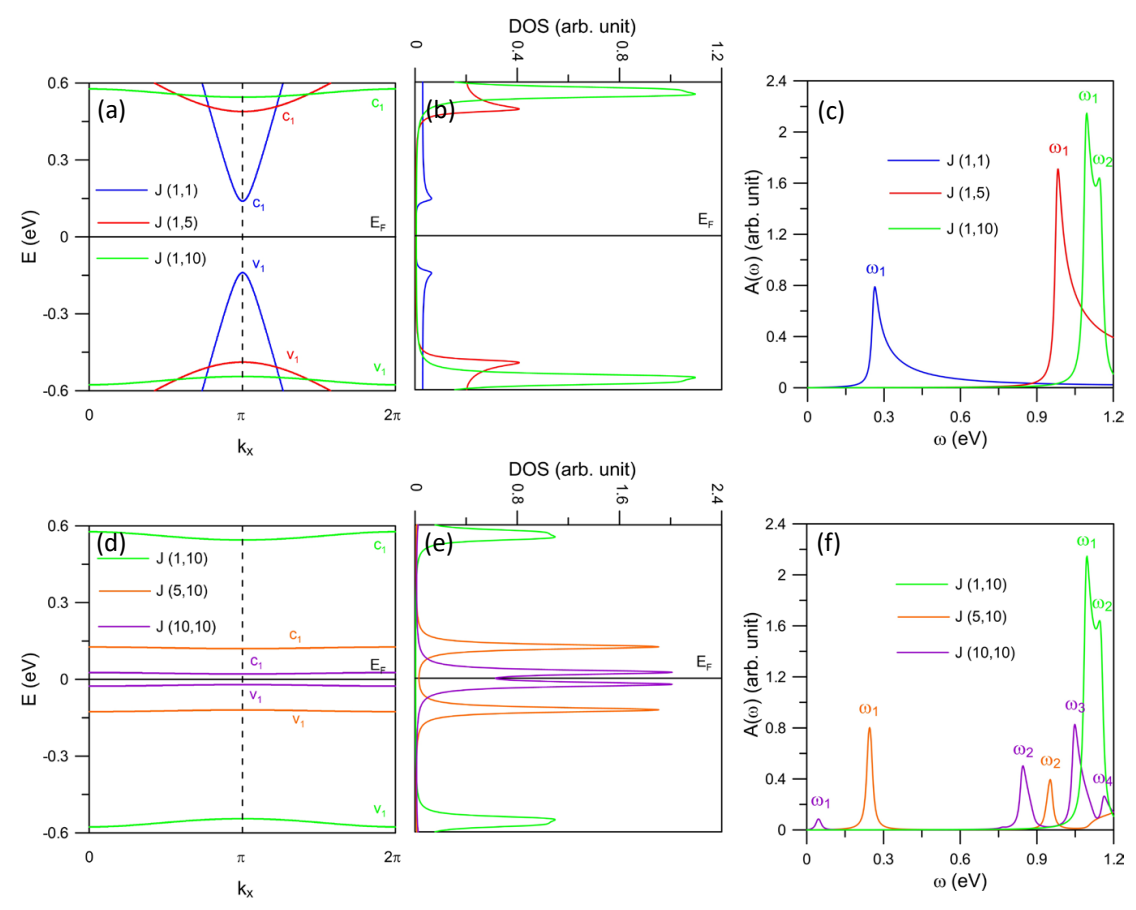

FIG. 2. (color online) (a) Electronic band structure of J-(n,m) AGNRs with $n=1$ and various chosen values of $m$, (b) the corresponding DOS and (c) the absorption spectra. Panels (d) through (f) illustrate similar plots for $m=10$ and various $n$ 's.

the band gap within the conduction states (for example, $c_{1}$ and $c_{2}$ ) or valence states (for example, $v_{1}$ and $v_{2}$ ), as shown in Fig. 3(a). This forms the double-peaks of the DOS (red lines in Fig. 3(b)) and also the absorption spectrum (red lines in Fig. 3(c)) for the case S-(1,5). Actually, this behavior also exists in the S-(1,10) case but with smaller spacing between the two components of the double-peaks (green lines). It is predicted that for the precise choice of $(n, m)$, the double-peaks might merge together to form a single structure with enhanced amplitude. However, even for the cases of S- $(5,10)$ and S- $(10,10)$ when $v_{1}$ and $v_{2}$ (also $c_{1}$ and $c_{2}$ ) mostly overlap (see Fig. $3(\mathrm{~d})$ ), the aforementioned optical selection rule results in suppression of the $\omega_{1}$ absorption intensity (see Fig. 3(f)).

Another optical selection rule is realized for the $\mathrm{S}-(n, m)$ case, which disallows the $v_{1} \rightarrow c_{2}$ and $v_{2} \rightarrow c_{1}$ transitions. It is noticed that, the unoccupied and occupied bands belonging to the forbidden excitation channels exhibit the same slopes. Such a phenomenon is related to their wave functions which determine the optical selection rule for the system. Explicitly, 
the special correlation between wave functions of the initial $\left|\Psi^{v}(\mathbf{k}, i)\right\rangle$ and final $\left|\Psi^{c}(\mathbf{k}, j)\right\rangle$ excitation states in Eq. (2) in the cases S- $(1,5)$ and $\mathrm{S}-(1,10)$ gives rise to certain forbidden optical transitions. The fact that the S- $(n, m)$ AGNR is subject to two different optical selection rules is attributed to its special energy dispersion due to the asymmetry of two ribbon sides.
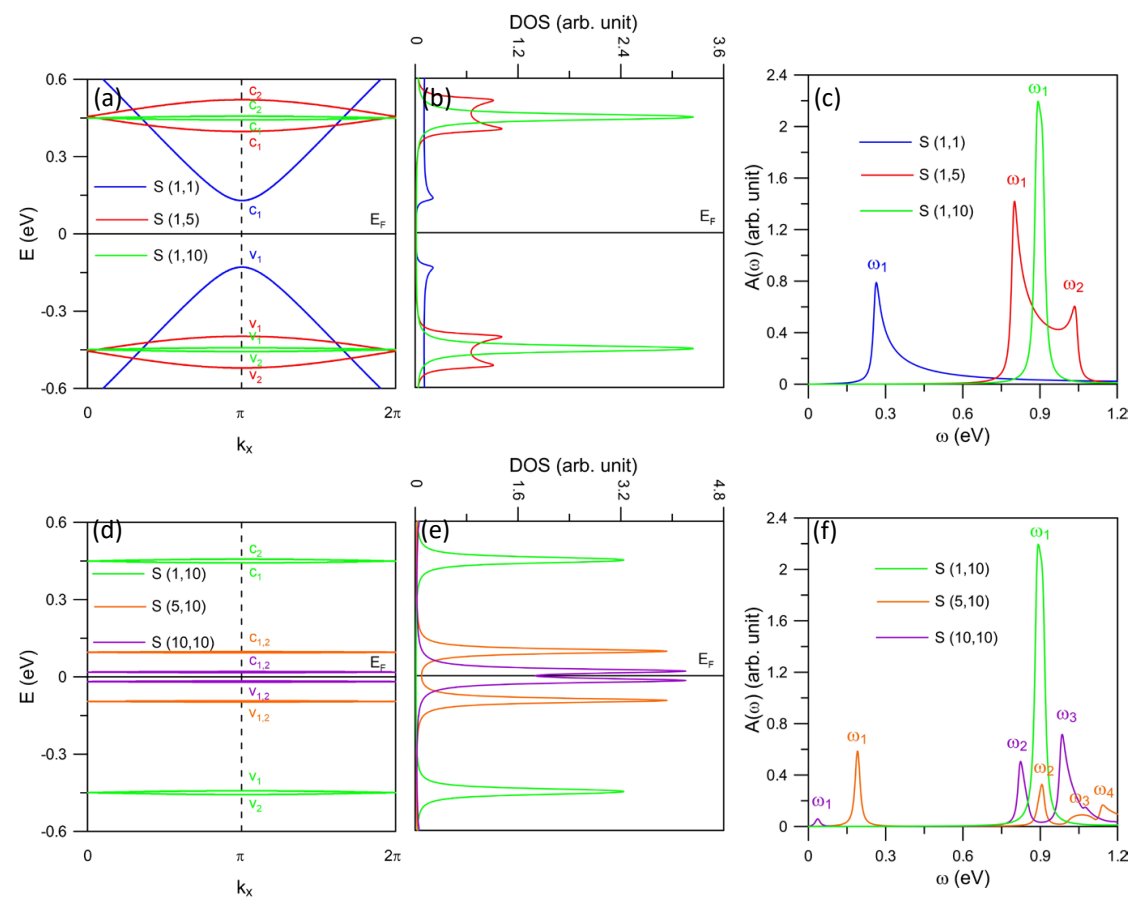

FIG. 3. (color online) (a) Electronic structure of S- $(n, m)$ AGNRs with $n=1$ and various $m$ 's. (b) The corresponding DOS and (c) absorption spectra. Panels (d) through (f) present similar plots for $m=10$ and various $n$ 's.

The edge-extended AGNRs with I- $(n, m)$ configuration present a distinctive energy dispersion and absorption spectra. In Fig. 4(a), the reformation of band structure follows the same way as for the $\mathrm{J}-(n, m)$ and $\mathrm{S}-(n, m)$ systems. Though, the energy spacing between the first $\left(v_{1}, c_{1}\right)$ and second $\left(v_{2}, c_{2}\right)$ pairs is quite large throughout the first Brillouin zone, which we refer to the purple lines in Fig. 4(d) for I- $(10,10)$. Furthermore, there exist the double-peak structures for I-(1,5), coming from the multi-extreme points of $v_{1}$ and $c_{1}$ at $k_{x}=0, \pi, 2 \pi$. This is different from the double-peak initiation of S- $(1,5)$ where the two component peaks correspond to two separated energy bands. 
Interestingly, the breakdown of the conventional optical selection rules discussed above is found in the I- $(n, m)$ AGNRs. It is straightforward that the $\left(v_{1}, c_{1}\right)$ pair becomes more flat for I-(1,5) (red lines in Fig. 4(a)) than for I-(1,1) (blue lines), Moreover, the corresponding DOS is higher for I-(1,5) (see Fig. 4(b)). However, as illustrated in Fig. 4(c), the threshold absorption peaks of I- $(1,5)$ are lower than that of I- $(1,1)$. We observe that the extraordinary $\mathrm{H}$ matrix elements originated from the unique lattice configuration of I- $(n, m)$ leading to such an unpredicted optical features. Additionally, the optical excitations between the nearly-flat bands give rise to substantial absorption peaks, for example, the $v_{2} \rightarrow c_{2}$ of the I- $(10,10)$ system (purple line in Fig. 4(f)). These behaviors are contrary to the cases of J- $(n, m)$ and S- $(n, m)$ discussed above, they do not obey the aforementioned selection rule which is based on the relationship between the wave functions. We note that both the optical selection rules are only applicable for the first pairs of edge bands. Our observation of such a singular optical characteristic is considered as a hint for the possible explanation of similar results from spectroscopy measurements.
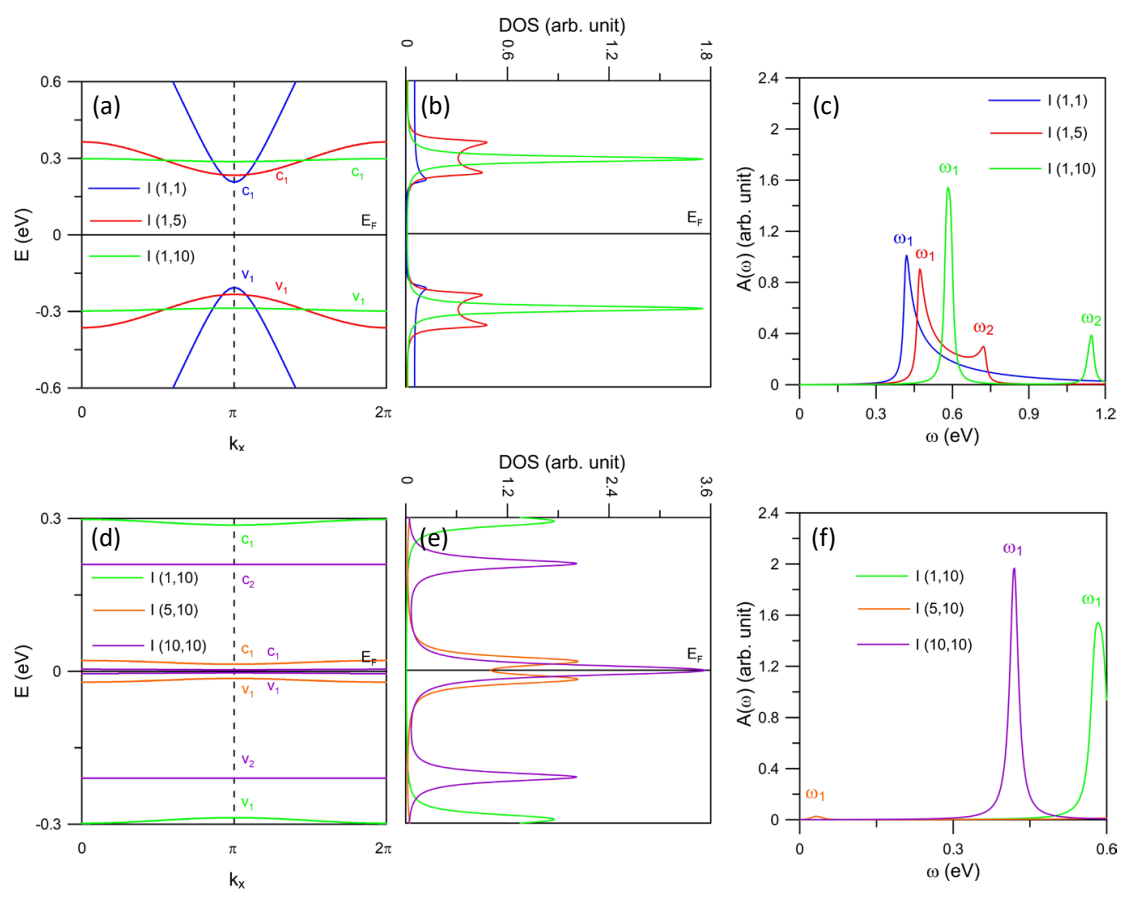

FIG. 4. (color online) (a) Band structure of I- $(n, m)$ AGNRs with $n=1$ and various $m$ 's. (b) The corresponding DOS and (c) absorption spectra. Panels (d) through (f) present similar plots for $m$ $=10$ and various $n$ values. 
Now we turn our attention to investigating the role played by the type of edge-extension in the electronic and absorption spectra of AGNRs. This can be understood via the comparison between the three different types of edge defects in terms of band structure, DOS, and absorption spectra. Due to special lattice asymmetric arrangement, the S- $(n, m)$ type of edge defect can adjust the band degeneracy while this is absent for the $\mathrm{J}-(n, m)$ and I- $(n, m)$ configurations. This explains the relatively higher DOS for S- $(n, m)$. The low-frequency absorption spectra present comparable absorption intensities regardless of the difference in DOS. In general, proper modification of $(n, m)$ can alter notably both the optical threshold and absorption intensity of the edge-extended AGNRs. The theoretical prediction offered in this work provides useful information in the search for suitable materials possible technology applications.

\section{CONCLUDING REMARKS}

In summary, we have investigated the rich electronic and optical properties of edgeextended AGNRs. The tight-binding Hamiltonian was constructed for each ribbon system with specific boundary conditions to study the band structures and DOS. The absorption spectra were computed from the absorption function. We found that these essential properties of AGNRs are remarkably enriched by modification of the edge states. The flat bands, band gap variation, and unique features of the DOS and absorption spectra were presented for various types and periods of the edge extension. Moreover, the unusual optical selection rule was demonstrated via the unusual transition channels associated with the flat bands for selected edge-modified systems. We showed that proper modification of $(n, m)$ can alter significantly both the optical threshold and absorption intensity of the edge-extended AGNRs. Our theoretical prediction opens an opportunity for extensive comprehension of the influence of edge defects on the electronic and optical properties of AGNRs.

Possible applications of nanoribbons could be in sensing and nano-imaging in very large wavelength range, extending from visible to infrared frequencies. [30, 31] Additionally, plasmons have been studied considerably in graphene $[32,33]$ and other bulk Dirac materials. Now, after almost two decades of unrelenting perseverance, [34, 35] the plasmon dynamical properties of low-dimensional structures are now well understood with the help of manyparticle theory. The polarization function can now be accurately calculated and plays a 
key role in calculations of the dielectric function which can in turn be used for determining plasmon dispersion and screening. Our results, on the other hand, reveal unique optical properties capable of generating significant interest. These nanomaterials are capable of hosting extremely strong light-matter interactions as a consequence of the enhanced excitonic effect in two dimensions. Therefore, it is crucial to fully understand the excitons to unlocking the potential of these nanoribbons for future photonic and optoelectronic devices. Finally, possible devices include optical modulators, excitonic light emitting diodes, lasers, and coupling in an optical cavity.

\section{ACKNOWLEDGMENTS}

T. N. Do would like to thank the SATU Presidents' Forum and the NCKU 90 and Beyond project for the support. P. H. Shih would like to thank the Ministry of Science and Technology of Taiwan for the support through the Grant No. MOST 110-2636-M-006002. G.G. would like to acknowledge the support from the Air Force Research Laboratory (AFRL) through Grant No. FA9453-21-1-0046. D.H. would like to acknowledge the financial support from the Air Force Office of Scientific Research (AFOSR).

[1] K. S. Novoselov, A. K. Geim, S. V. Morozov, D. Jiang, Y. Zhang, S. V. Dubonos, I. V. Grigorieva, and A. A. Firsov, Electric Field Effect in Atomically Thin Carbon Films, Science 306,666 (2004).

[2] Y. Li, W. Zhang, M. Morgenstern, and R. Mazzarello, Electronic and Magnetic Properties of Zigzag Graphene Nanoribbons on the (111) Surface of Cu, Ag, and Au, Phys. Rev. Lett. 110, 216804 (2013).

[3] T.-N. Do, P.-H. Shih, G. Gumbs, and D. Huang, Influence of Electric and Magnetic Fields and $\sigma$-Edge Bands on the Electronic and Optical Spectra of Graphene Nanoribbons, Phys. Rev. B 103, 115408 (2021).

[4] L. G. Cancado, M. A. Pimenta, B. R. A. Neves, G. Medeiros-Ribeiro, T. Enoki, Y. Kobayashi, K. Takai, K. Fukui, M. S. Dresselhaus, R. Saito, and A. Jorio, Anisotropy of the Raman Spectra of Nanographite Ribbons, Phys. Rev. Lett. 93, 047403 (2004). 
[5] A. I. Chernov, P. V. Fedotov, A. V. Talyzin, I. Suarez Lopez, I. V. Anoshkin, A. G. Nasibulin, E. I. Kauppinen, and E. D. Obraztsova, Optical Properties of Graphene Nanoribbons Encapsulated in Single-Walled Carbon Nanotubes, ACS Nano 7, 6346 (2013).

[6] K. Kusakabe and M. Maruyama, Magnetic Nanographite, Phys. Rev. B 67, 092406 (2003).

[7] K. Tada and K. Watanabe, Ab Initio Study of Field Emission from Graphitic Ribbons, Phys. Rev. Lett. 88, 127601 (2002).

[8] Z. Xu, Q.-S. Zheng, and G. Chen, Elementary Building Blocks of Graphene-Nanoribbon-Based Electronic Devices, Appl. Phys. Lett. 90, 223115 (2007).

[9] D. Soriano, F. Munoz-Rojas, J. Fernandez-Rossier, and J. J. Palacios, Hydrogenated Graphene Nanoribbons for Spintronics, Phys. Rev. B 81, 165409 (2010).

[10] S. Zamani and R. Farghadan, Graphene Nanoribbon Spin-Photodetector, Phys. Rev. Applied 10, 034059 (2018).

[11] J. Cai, P. Ruffieux, R. Jaafar, M. Bieri, T. Braun, S. Blankenburg, M. Muoth, A. P. Seitsonen, M. Saleh, X. Feng, K. Mullen, and R. Fasel, Atomically Precise Bottom-up Fabrication of Graphene Nanoribbons, Nature 466, 470 (2010).

[12] Y.-C. Chen, T. Cao, C. Chen, Z. Pedramrazi, D. Haberer, D. G. de Oteyza, F. R. Fischer, S. G. Louie, and M. F. Crommie, Molecular Bandgap Engineering of Bottom-up Synthesized Graphene Nanoribbon Heterojunctions, Nature Nanotechnology 10, 156 (2015).

[13] M. Mehdi Pour, A. Lashkov, A. Radocea, X. Liu, T. Sun, A. Lipatov, R. A. Korlacki, M. Shekhirev, N. R. Aluru, J. W. Lyding, V. Sysoev, and A. Sinitskii, Laterally Extended Atomically Precise Graphene Nanoribbons with Improved Electrical Conductivity for Efficient Gas Sensing, Nature Communications 8, 820 (2017).

[14] D. J. Rizzo, G. Veber, T. Cao, C. Bronner, T. Chen, F. Zhao, H. Rodriguez, S. G. Louie, M. F. Crommie, and F. R. Fischer, Topological Band Engineering of Graphene Nanoribbons, Nature 560, 204 (2018).

[15] Q. Sun, X. Yao, O. Groning, K. Eimre, C. A. Pignedoli, K. Mullen, A. Narita, R. Fasel, and P. Ruffieux, Coupled Spin States in Armchair Graphene Nanoribbons with Asymmetric Zigzag Edge Extensions, Nano Letters 20, 6429 (2020).

[16] M. Y. Han, B. Ozyilmaz, Y. Zhang, and P. Kim, Energy Band-Gap Engineering of Graphene Nanoribbons, Phys. Rev. Lett. 98, 206805 (2007).

[17] L. Tapaszto, G. Dobrik, P. Lambin, and L. P. Biro, Tailoring the Atomic Structure of Graphene 
Nanoribbons by Scanning Tunnelling Microscope Lithography, Nature Nanotechnology 3, 397 (2008).

[18] D. V. Kosynkin, A. L. Higginbotham, A. Sinitskii, J. R. Lomeda, A. Dimiev, B. K. Price, and J. M. Tour, Longitudinal Unzipping of Carbon Nanotubes to Form Graphene Nanoribbons, Nature 458, 872 (2009).

[19] G. Z. Magda, X. Jin, I. Hagymasi, P. Vancso, Z. Osvath, P. Nemes-Incze, C. Hwang, L. P. Biro, and L. Tapaszto, Room-Temperature Magnetic Order on Zigzag Edges of Narrow Graphene Nanoribbons, Nature 514, 608 (2014).

[20] T. Wang, Z. Wang, R. V. Salvatierra, E. McHugh, and J. M. Tour, Top-down Synthesis of Graphene Nanoribbons Using Different Sources of Carbon Nanotubes, Carbon 158, 615 (2020).

[21] P. Ruffieux, J. Cai, N. C. Plumb, L. Patthey, D. Prezzi, A. Ferretti, E. Molinari, X. Feng, K. Mullen, C. A. Pignedoli, and R. Fasel, Electronic Structure of Atomically Precise Graphene Nanoribbons, ACS Nano 6, 6930 (2012).

[22] L.-J. Shi, L.-Z. Yang, J.-Q. Deng, L.-H. Tong, Q. Wu, L. Zhang, L. Zhang, L.-J. Yin, and Z. Qin, Constructing Graphene Nanostructures with Zigzag Edge Terminations by Controllable STM Tearing and Folding, Carbon 165, 169 (2020).

[23] O. Groning, S. Wang, X. Yao, C. A. Pignedoli, G. Borin Barin, C. Daniels, A. Cupo, V. Meunier, X. Feng, A. Narita, K. Mullen, P. Ruffieux, and R. Fasel, Engineering of Robust Topological Quantum Phases in Graphene Nanoribbons, Nature 560, 209 (2018).

[24] T. Cao, F. Zhao, and S. G. Louie, Topological Phases in Graphene Nanoribbons: Junction States, Spin Centers, and Quantum Spin Chains, Phys. Rev. Lett. 119, 076401 (2017).

[25] J.-P. Joost, A.-P. Jauho, and M. Bonitz, Correlated Topological States in Graphene Nanoribbon Heterostructures, Nano Lett. 19, 9045 (2019).

[26] T.-N. Do, P.-H. Shih, G. Gumbs, and D. Huang, Engineering Plasmon Modes and Their Loss in Armchair Graphene Nanoribbons by Selected Edge-Extended Defects, J. Phys.: Condens. Matter 33, 485001 (2021).

[27] P.-H. Shih, T.-N. Do, B.-L. Huang, G. Gumbs, D. Huang, and M.-F. Lin, Magneto-Electronic and Optical Properties of Si-Doped Graphene, Carbon 144, 608 (2019).

[28] Kohmoto, M. Topological Invariant and the Quantization of the Hall Conductance. Annals of Physics 1985, 160, 343-354.

[29] Y.-W. Son, M. L. Cohen, and S. G. Louie, Energy Gaps in Graphene Nanoribbons, Phys. Rev. 
Lett. 97, 216803 (2006).

[30] Kaveh Khaliji, Sudipta Romen Biswas, Hai Hu, Xiaoxia Yang, Qing Dai, Sang-Hyun Oh, Phaedon Avouris, and Tony Low, Plasmonic Gas Sensing with Graphene Nanoribbons, Physical Review Applied 13, 011002 (2020).

[31] F. Hu, Y. Luan, Z. Fei, I. Z. Palubski, M. D. Goldflam, S. Dai, J.-S. Wu, K. W. Post, G. C. A. M. Janssen, M. M. Fogler, and others, Imaging the localized plasmon resonance modes in graphene nanoribbons, Nano letters 17, 5423-5428 (2017).

[32] Alexander N. Grigorenko, Marco Polini, and K. S. Novoselov, Graphene plasmonics, Nature photonics 6, 749-758 (2012).

[33] F. Javier Garcia de Abajo, Graphene plasmonics: challenges and opportunities,Acs Photonics 1, 135-152 (2014).

[34] E. H. Hwang and S. Das Sarma, ,Dielectric function, screening, and plasmons in twodimensional graphene, Physical Review B 75, 205418 (2007).

[35] P. K. Pyatkovskiy, Dynamical polarization, screening, and plasmons in gapped graphene, Journal of Physics: Condensed Matter 21, 025506 (2008). 